\title{
Multilinguales
}

1 | 2013

Pratiques littéraires, linguistiques, pédagogiques,

didactiques et médiations culturelles contemporaines

\section{Compétences initiales et transmission des langues secondes et étrangères au Cameroun}

Initial Competences and the Transmission of Second and Foreign Languages Case of Cameroon

\section{Julia Ndibnu Messina Ethe}

\section{(2) OpenEdition}

Journals

Édition électronique

URL : https://journals.openedition.org/multilinguales/3199

DOI : $10.4000 /$ multilinguales.3199

ISSN : 2335-1853

Éditeur

Université Abderrahmane Mira - Bejaia

Édition imprimée

Date de publication : 1 juin 2013

Pagination : 105-119

ISSN : 2335-1535

Référence électronique

Julia Ndibnu Messina Ethe, " Compétences initiales et transmission des langues secondes et étrangères au Cameroun », Multilinguales [En ligne], 1 | 2013, mis en ligne le 01 juin 2013, consulté le 21 septembre 2021. URL : http://journals.openedition.org/multilinguales/3199 ; DOI : https://doi.org/ 10.4000/multilinguales.3199

Ce document a été généré automatiquement le 21 septembre 2021

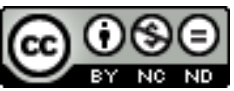

Multilinguales est mise à disposition selon les termes de la Licence Creative Commons Attribution Pas d'Utilisation Commerciale - Pas de Modification 4.0 International 


\title{
Compétences initiales et transmission des langues secondes et étrangères au Cameroun
}

\author{
Initial Competences and the Transmission of Second and Foreign Languages Case \\ of Cameroon
}

Julia Ndibnu Messina Ethe

1 Sous protectorat allemand, cédé ensuite à La France et au Royaume-Uni, le Cameroun présente une très grande variété de langues et de cultures. Il existe près de 248 langues autochtones et régionales (Dieu et Renaud, 1983 ; Bitjaa Kody, 2004), aux côtés de deux langues officielles, le français et l'anglais, et deux langues issues de leur contact, le camfranglais et le pidgin.

2 Dans un environnement de formation " plurilingue ${ }^{1}$ ", et compte tenu des compétences linguistiques asymétriques des apprenants, les autorités éducatives du Cameroun ont reparti les écoles de formation des enseignants en deux grands cycles : le primaire et le supérieur. Les écoles des instituteurs concernées par l'enseignement des langues secondes, à savoir l'anglais et le français, excluent les langues régionales des programmes. Le cycle supérieur s'occupe de la formation des enseignants des lycées. Ils sont formés à l'enseignement du français, de l'anglais et des langues régionales. Les langues régionales sont intégrées au secondaire depuis 2008. Ce succès est redevable au Programme Opérationnel pour l'enseignement des langues au Cameroun (PROPELCA), mis sur pied par l'Université de Yaoundé et l'Association Nationale des Comités de Langues Camerounaises (ANACLAC), depuis 1982.

3 Les langues française et anglaise évoluent et se régionalisent : le français a donné lieu à une nouvelle variante le camfranglais ${ }^{2}$, l'anglais a engendré le pidgin-english ${ }^{3}$ (Biloa, 2003). Ces deux variantes altèrent les performances et les compétences communicatives langagières de l'ensemble des apprenants : élèves, étudiants et futurs professeurs. Mais les cours de français, d'anglais et des langues régionales ne tiennent pas compte de cette donnée dans les contenus des enseignements et les tâches proposées aux 
apprenants. Comment transmettre le savoir sans envisager ces préalables? Quels sont les principes maîtres d'une bonne gestion des séances de cours de L2?

4 Cet article mettra l'accent sur les statuts des langues enseignées au Cameroun, la présentation des écoles de formation et des objectifs pédagogiques opérationnels qui conduisent à une formation initiale exemplaire en L2.

\section{Statuts des langues au Cameroun}

5 Au Cameroun, les statuts des langues sont de plusieurs ordres. Nous n'en retiendrons que quatre, en nous référant à la définition d'Adopo et al. (1997): «[Le statut est] l'ensemble des données juridiques, politiques et économiques relatives à la langue. Ces données nous permettent de déterminer l'importance sociale de la langue » (13).

6 L'anglais et le français, langues officielles, sont susceptibles de coexister avec des langues régionales, des langues secondes et des langues maternelles (LM).

7 Les maternelles représentent éventuellement des langues véhiculaires, des langues régionales et surtout, comme dans cet article, des langues secondes. Pour assumer des rôles véhiculaires, à l'exemple des langues régionales, il faut que les LM jouissent d'une reconnaissance avérée, d'un nombre de locuteurs élevé et d'études scientifiques (Fishman, 1976). Le schéma suivant dépeint la stratification des langues au Cameroun.

Figure 1 : différents groupes de langues au Cameroun

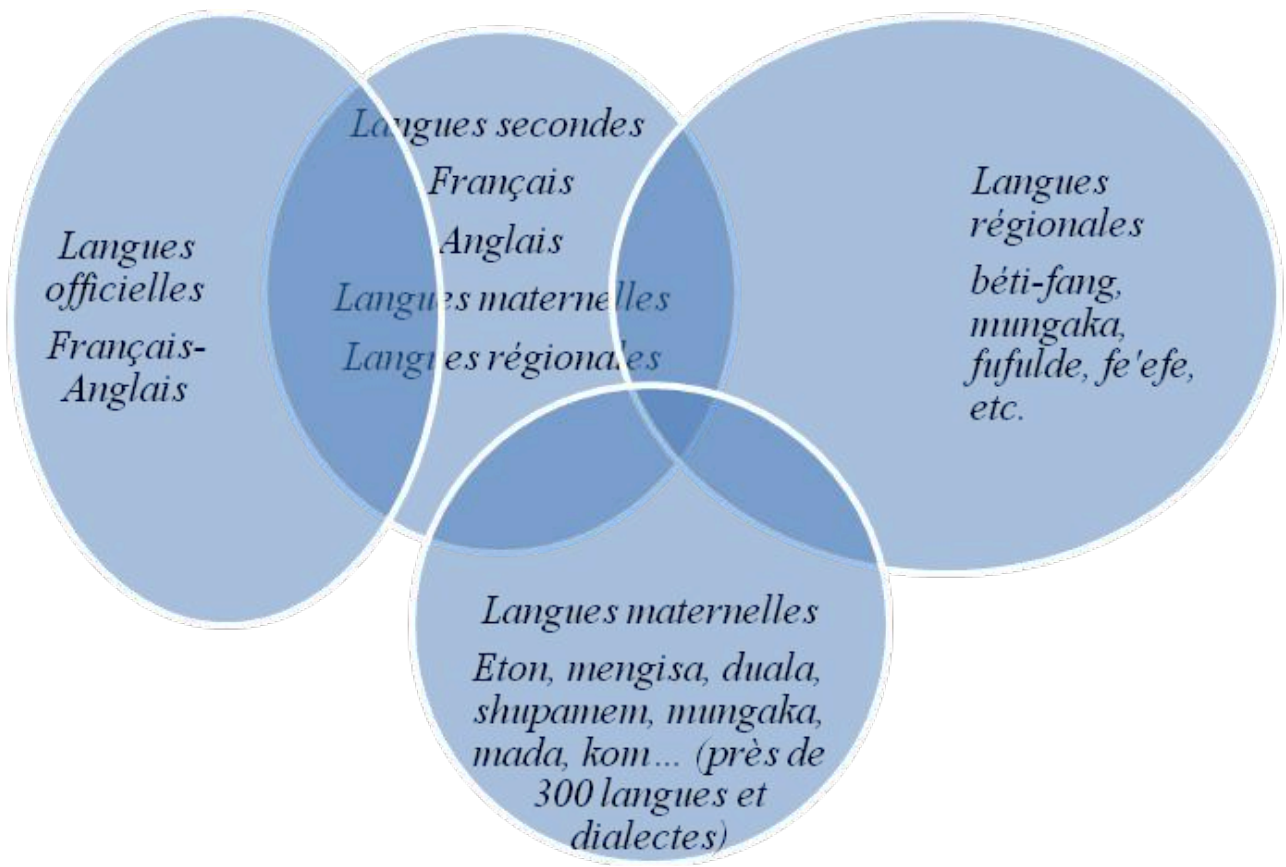

Les intersections indiquent les connexions existant entre les différents groupes, comme les éclaircissements ci-dessous le relèveront.

A) Les langues officielles : Shell et Wiesemann (2000:39) assurent que les langues officielles sont «la ou les langue(s) européenne(s) utilisée(s) pour les affaires du gouvernement et aussi comme langue(s) principale(s) de l'éducation ». Pour Adopo et al. (1997: 13), les langues officielles incarnent»les langues qu'un État utilise pour l'ensemble de son fonctionnement aux différents niveaux (administration, justice, 
armée, éducation, activités nationales et internationales ». Le système éducatif en Afrique subsaharienne adopte généralement les langues officielles en tant que langues de certaines disciplines et langues d'enseignement. C'est le cas au Gabon, au Tchad et au Cameroun avec le français. Le Cameroun se singularise par son bilinguisme avec des sections qui offrent des enseignements dans lesdites langues, en tant que langues secondes, aussi bien dans les grandes écoles de formation que dans les écoles primaires et secondaires.

B) Les langues régionales : au Cameroun, les langues régionales représentent les langues auxquelles le gouvernement a octroyé un statut officiel, celui de langues nationales. Les langues régionales correspondent aux langues de grande communication au sein du pays et dans des régions précises à savoir le duala dans la Région du Littoral, le beti-fang dans les régions du Sud et du Centre, le fulfulde dans les régions du Nord et de l'Extrême-Nord. Considérées comme langues secondes depuis 2008, elles sont enseignées dans les établissements du secondaire.

11 C) Les langues secondes: la langue seconde est celle que les locuteurs utilisent quotidiennement dès qu'ils sont en âge d'être scolarisés, dans le commerce, les communications interpersonnelles incluant des langues et des cultures régionales différentes. Elle est apprise à la suite de la langue première. Aussi, les créoles telles que le pidgin-english et le camfranglais sont considérés comme des langues secondes au même titre que le français et l'anglais en milieu monolingue rural.

D) Les langues maternelles : la langue maternelle est celle que l'enfant acquiert et utilise en famille. Son statut se limite à la cellule familiale ou clanique. C'est par elle que l'éducation traditionnelle est transmise aussi bien dans les villages que dans les zones urbaines. Le pidgin-english et le français deviennent au fil des ans des langues premières (ou maternelles) des jeunes qui deviendront plus tard des enseignants de langues secondes. Ils maîtrisent partiellement leurs langues maternelles et les langues à enseigner. Les écoles de formation sont en principe chargées de renforcer les savoirs acquis en français, anglais et langues régionales. Elles se doivent dès lors de fixer des objectifs susceptibles de combler les attentes des apprenants. Mais, au vu de tous les groupes de langues sur le territoire camerounais, il est pertinent de savoir quels sont les objectifs pédagogiques opérationnels que se fixent les Écoles de formation pour l'enseignement des L2.

\section{Ecoles de formation et objectifs d'enseignement}

Il existe plusieurs écoles professionnelles destinées à la formation des élèves enseignants à savoir les ÉNIEG (École Normale des Instituteurs de l'Enseignement Général), les ÉNIET (École Normale des Instituteurs de l'Enseignement Technique) pour les élèves maitres, l'ENS (École Normale Supérieure) et l'ENSET (École Normale Supérieure de l'Enseignement Technique) pour les élèves professeurs.

14 A) École normale des instituteurs de l'enseignement général (ENIEG) : l'ÉNIEG forme des enseignants pour le cycle primaire. Les objectifs linguistiques poursuivis au cours de la formation des élèves - instituteurs de l'enseignement général sont :

- permettre aux futurs maîtres d'acquérir une meilleure maîtrise du français (pour les francophones) et de l'anglais (pour les anglophones) pour mieux transmettre les savoirfaire en grammaire, conjugaison, vocabulaire, rédaction. 
- initier les élèves enseignants à la transmission des données orales en français, en anglais, en langues régionales actuellement enseignées (fufulde, mungaka, duala, basaa, beti-fang) afin que leurs écoliers puissent communiquer oralement quel que soit le milieu environnant.

- recourir aux approches pédagogiques en vigueur, à savoir la Nouvelle Approche Pédagogique, l'Approche par Compétence et l'approche par l'action.

B) École normale des instituteurs de l'enseignement technique (ENIET) : déceler les objectifs de la formation des élèves instituteurs nous paraît difficile. Au primaire, les deux seules activités techniques contenues dans les programmes d'enseignement au primaire (2000) sont le travail manuel et les activités pratiques. Il ne comporte aucun cours de langue, ni première ni seconde.

$\mathrm{Au}$ sortir de leur formation, les élèves instituteurs enseignent dans les écoles primaires d'enseignement général. Aucune performance préliminaire en langue ne leur est exigée, excepté au cours du concours d'entrée en sixième qui comprend une épreuve de dictée-questions ou de compréhension de texte en français et en anglais. Les langues maternelles en tant que langues secondes sont absentes tout au long de leur formation.

c) École normale supérieure (ENS) : les ENS ont en charge la formation des élèves professeurs pour leur intégration dans le secondaire. Les objectifs visés sont :

- développer chez les élèves professeurs la capacité à enseigner la grammaire, le vocabulaire, la rhétorique, la compréhension des textes, la dissertation.

- initier l'élève professeur à utilisation des approches pédagogiques en vigueur, à savoir la Nouvelle Approche Pédagogique, l'Approche par Compétence et l'approche par l'action.

18 D) Ecole normale supérieure de l'enseignement technique (ENSET) : plusieurs Écoles normales supérieures d'enseignement technique ont pour mission la formation des élèves professeurs en technique et en technologie.

19 À l'ENSET, aucune spécialité ou filière n'est réservée à l'enseignement des langues. Il est l'objet d'une unité de valeur intitulée «Formation Bilingue» (le français aux anglophones, l'anglais aux francophones), pour les L2, trois heures par semaine et à moins de quatre mois par an. Les objectifs de ces ENSET sont :

- former les élèves professeurs à enseigner dans les deux langues officielles (français et anglais), dans tous les établissements d'enseignement technique du Cameroun.

- leur inculquer le vocabulaire technique approprié pour l'enseignement des matières technologiques.

- les initier aux trois approches pédagogiques citées plus haut.

Les étudiants professeurs considèrent leurs futurs élèves comme des locuteurs natifs des langues nationales (L2), du français et de l'anglais. Comment mettre en œuvre une formation visant les objectifs précités?

\section{Enquête et informateurs}

21 La population étudiée rassemble les acteurs de l'enseignement : les instructeurs et les apprenants. Les tableaux exposent les résultats des déclarations des sondés au cours de l'année 2011. Les tableaux subséquents indiquent les écoles sollicitées, le nombre d'interrogés et les langues des enquêtés. 
Tableau 1 : langues parlées par les enseignants

\begin{tabular}{|l|l|l|}
\hline Écoles & Nombre d'enquêtés & Langues parlées par les enseignants \\
\hline ÉNIEG & 01 & français, anglais, LM \\
\hline ENS de Yaoundé I & 02 & français, anglais, LM, pidgin \\
\hline ENSET & 02 & français, anglais \\
\hline Total & 05 & français, anglais, LM, pidgin, \\
\hline
\end{tabular}

Tableau 2 : langues parlées par les apprenants

\begin{tabular}{|c|c|c|}
\hline Écoles & Nombre d'enquêtés & Langues parlées par les enseignants \\
\hline ÉNIEG & 15 & \multirow{3}{*}{$\begin{array}{l}\text { français, anglais, LM, pidgin } \\
\text { LN, camfranglais }\end{array}$} \\
\hline ENS de Yaoundé I & 10 & \\
\hline ENSET de Douala & 30 & \\
\hline Total & 65 & français, anglais, LM, pidgin, \\
\hline
\end{tabular}

Dans cette étude, l'approche genre n'a pas été retenue, car le but premier du présent article est la détermination des approches pédagogiques et des différentes matières linguistiques à enseigner aux élèves professeurs.

23 Soixante-dix personnes représentent l'ensemble des volontaires auprès desquels les investigations ont été menées. Il est à noter que l'ENSET figure ici pour sa consolidation des cours de LO2 et l'intégration dans les programmes, des cours de langue spécialisée et de langue nationale.

24 Les enseignants sondés maîtrisent principalement le français, l'anglais, les LM et le pidgin. L'observation établit que les formateurs des formateurs manient plusieurs langues, mais prohibent les parlers argotiques comme le camfranglais et parfois le pidgin. Les apprenants quant à eux utilisent les langues indépendamment de l'interlocuteur, mais ils plébiscitent la langue la mieux maîtrisée pour leur spécialisation pédagogique.

Pour transmettre ou enseigner les langues secondes, les deux groupes en présence font face à des difficultés de plusieurs ordres. Au niveau des apprenants, on peut détecter des problèmes phonétiques et morphosyntaxiques, par exemple. Les enseignants, quant à eux, éprouvent des difficultés relatives aux approches pédagogiques utilisées pour enseigner. 


\section{Difficultés des apprenants en anglais, en français et en langue nationale}

Les apprenants de langues nationales, de français et d'anglais, rencontrent plusieurs types de difficultés. Elles se résument en l'agrammaticalité des phrases, l'absence de maîtrise des concepts et phénomènes culturels, la non maîtrise des évolutions et des approches pédagogiques.

A) L'agrammaticalité des phrases à l'oral et à l'écrit : l'agrammaticalité repose sur les différentes manipulations syntaxiques opérées sur des mots ou des groupes de mots. Par moments, l'analyse de ces manipulations conduit à une détection des erreurs identiques et répétitives chez les apprenants des L2. Des énoncés recueillis, nous observons la récurrence de deux manipulations syntaxiques à savoir l'effacement et la substitution.

Les élèves professeurs tendent à omettre la marque du temps à l'oral et surtout à l'écrit. Cette omission a des conséquences au niveau tonal et quelquefois lexical. C'est le cas des énoncés des apprenants de première année à l'ENS de Yaoundé I, dans l'exemple cidessous :

Exemple (1) a : en duálá/ bàsàá

\begin{tabular}{|l|l|}
\hline $\begin{array}{l}\text { Enoncé de l'apprenant } \\
\text { * Nà dá dìkúbè }\end{array}$ & $\begin{array}{l}\text { Enoncé approprié } \\
\text { Nà mà.dá dìkúbé }\end{array}$ \\
je manger banane & je prés.mange banane \\
je mange une banane & je mange une banane \\
\hline
\end{tabular}

On constate la disparition de "mà " qui marque le présent de l'indicatif, et le non respect des tonèmes, le ton bas remplaçant souvent le ton haut. Les phonèmes inexistants dans la langue première, comme « $6, d$, sont substitués par « $b, d »$. Ces cas d'effacement et de substitution sont fréquents chez les apprenants de première année.

Les apprenants de l'ENSET de Douala I et de l'ENIEG, quant à eux, insèrent des morphèmes de pidgin dans les énoncés en anglais.

Exemple (1) b : en anglais

\begin{tabular}{|c|c|}
\hline Enoncé de l'apprenant & Enoncé approprié \\
\hline $\begin{array}{l}\text { * A dõ go skul tude } \\
\text { je prés. partir école aujourd'hui }\end{array}$ & $\begin{array}{l}\text { Ai gou tu skul tə'dei } \\
\text { je prés. à école aujourd'hui }\end{array}$ \\
\hline Je vais à l'école aujourd'hui & Je vais à l'école aujourd'hui \\
\hline $\begin{array}{l}\text { *A dõ t } \mathbf{t} \mathbf{o p} \text { ko :n } \\
\text { je prés. manger maïs }\end{array}$ & $\begin{array}{l}\text { Ai i :t ko :n } \\
\text { je mange maïs }\end{array}$ \\
\hline je mange du maïs & je mange du maïs \\
\hline
\end{tabular}


Dans les deux exemples, on constate l'effacement des diphtongues «ai, au, ei » et l'omission totale $\mathrm{du}$ « tu » après le verbe. Les apprenants introduisent un morphème de temps «dõ » qui marque le présent de l'indicatif en pidgin. Ils substituent les diphtongues «ai, au, ei » par «a, o, e », et le verbe « eat » par celui qu'ils maitrisent, « t $\int \mathrm{p}$ ». On constate que le pidgin corrompt l'anglais à travers des mots empruntés et l'effacement de plusieurs phones et morphèmes.

Exemple (1) c : en français

\begin{tabular}{|l|l|}
\hline Enoncé de l'apprenant & Enoncé approprié \\
*3ə Siba pur tJop & 3ə desã pur mãze \\
je descends pour manger & je descends pour manger \\
Je descends pour manger & Je descends pour manger \\
\hline
\end{tabular}

En français, le camfranglais modifie les propos des apprenants débutants, mais il manifeste plus de substitutions que d'effacements. Ces changements affectent le niveau des performances des élèves professeurs. La pratique constante du camfranglais et $d u$ pidgin-english en milieu institutionnel et en milieu naturel influe lourdement sur les attitudes à adopter dans les institutions formatives.

B) L'absence de maîtrise des implicites culturels du discours : Kerbrat-Orecchioni (1986: 25) considère comme implicite culturel: "toutes les informations qui, sans être ouvertement posées, sont cependant automatiquement entraînées par la formulation de l'énoncé, dans lequel elles se trouvent intrinsèquement inscrites, quelle que soit la spécificité du cadre énonciatif ».

Par exemple, «Pierre ne fume plus » présuppose dans l'énoncé même que Pierre fumait auparavant.

Quant aux sous-entendus, ce sont "toutes les informations qui sont susceptibles d'être véhiculées par un énoncé donné, mais dont l'actualisation reste tributaire de certaines particularités du contexte énonciatif ».

$\mathrm{Au}$ Cameroun ces implicites culturels se traduisent par la gestuelle et des phrases telles que :

Exemple (2) : en mengisa

\begin{tabular}{|l|}
\hline (1)Zùgá ó nyóng màndím má zóng \\
Viens tu prendre eau de zong \\
viens prendre de l'eau du zong \\
\hline (2) Mbàng ì tà.nó \\
Pluie il prés.pleuvoir \\
il pleut (il y a un peu d'argent) \\
\hline
\end{tabular}

En (1), c'est une promesse implicite sur la base d'un fruit très amer (màndím má zóng). Les apprenants doivent mettre en contexte leur vocabulaire pour maîtriser ce genre 
d'implicite. Au Cameroun, en français comme en LM, l'expression « il pleut» a une connotation d'abondance matérielle.

Exemple (3) : en français et en anglais :

Cette fois tu as gagné le gros lot.

Gagner le gros lot, selon les contextes peut signifier gagner de l'argent ou réussir à trouver un bon parti, par exemple un époux ou un partenaire d'entreprise... La maîtrise du contexte social guide l'interprétation des propos et la détection des ambiguïtés et des implicites.

c) L'absence de maîtrise de contenus culturels nécessaires en situation d'argumentation à l'oral et à l'écrit: les principaux contenus culturels non maîtrisés sont les proverbes, les maximes, les notions de respects aussi bien dans la gestuelle que dans le vocabulaire. Nous ne reviendrons pas sur les proverbes, mais sur les marques de respect souvent ignorées. Dans les langues camerounaises, le salut adressé à un aîné ou à un chef se distingue de celui destiné à une personne considérée comme son égale.

Dans les régions du Nord-Ouest et de l'Ouest, celui qui salue peut frapper des mains trois à sept fois au maximum. Au Nord, les interlocuteurs se palpent le bras, de la main à l'épaule, en sollicitant des réponses sur l'état de santé des membres de la famille.

En Angleterre, la politesse exige qu'on dise " you're welcome » ou « come again » à un hôtel quelle que soit l'humeur de celui qui le reçoit. Le Cameroun a la particularité de posséder plusieurs cultures, mais de nos jours, ignorées par les jeunes (Messina Ethé, 2010), et enseignées timidement au secondaire. Tous les contenus culturels comme la danse, les contes, les chants traditionnels, les berceuses font également partie du répertoire très peu connu des apprenants de L2.

D) La non maîtrise des approches didactiques en L2, FLE ou ALE ${ }^{4}$ et des approches pédagogiques récentes (l'approche par compétence, l'approche par l'action, l'approche audiovisuelle) : les apprenants ignorent les approches développées au cours de ces dernières années et qui prônent la centration sur l'apprenant. Or, en FLE, ALE et L2, la didactique est devenue plus participative.

42 Le tableau subséquent présente les pourcentages d'élèves professeurs qui déclarent exploiter les différentes approches d'enseignement des langues secondes et étrangères.

Tableau 3 : maîtrise des approches pédagogiques

\begin{tabular}{|l|l|l|l|l|l|}
\hline $\begin{array}{l}1-\text { Approche } \\
\text { Grammaire- } \\
\text { traduction }\end{array}$ & $\begin{array}{l}2 \\
\text { Approche } \\
\text { Audio- } \\
\text { visuelle }\end{array}$ & $\begin{array}{l}3-\text { Approche } \\
\text { culturelle }\end{array}$ & $\begin{array}{l}4-\quad \text { Nouvelle } \\
\text { Approche } \\
\text { Pédagogique }\end{array}$ & $\begin{array}{l}5 \text { - Approche par } \\
\text { compétence }\end{array}$ & $\begin{array}{l}6 \quad \text {-Approche } \\
\text { par l'action }\end{array}$ \\
\hline $100 \%$ & $40 \%$ & $25 \%$ & $80 \%$ & $50 \%$ & $25 \%$ \\
\hline
\end{tabular}

43 La première méthode d'enseignement est la plus connue et la plus répandue; la quatrième a été appliquée de 2000 à 2008 ; la cinquième (l'approche par compétence) est la plus récente. L'approche par l'action n'est connue que de $25 \%$ des apprenants et d'après eux, elle ne saurait satisfaire les besoins des élèves des classes de langues camerounaises. 

permettraient une transmission adéquate des connaissances, telle que préconisée par le CECR, à savoir la mise en relation des cultures et l'établissement du contact avec les personnes à travers une langue, ici, une L2 (Puren, 2006). Les approches demeurent centrées sur l'enseignant et non sur l'apprenant comme préconisé dans les approches par compétence et par l'action. Toutes ces difficultés empêchent les enseignants d'atteindre aisément leurs objectifs pédagogiques. Il leur est difficile de transmettre le savoir, le savoir-faire et le savoir-être. Pourtant, les problèmes rencontrés par les apprenants montrent qu'il est nécessaire de tenir compte de la centration sur l'apprenant en s'intéressant davantage à la notion d'interculturalité ${ }^{5}$ dans la formation des enseignants de langue.

\section{Difficultés rencontrées par les enseignants de langues}

La maîtrise des langues varie d'un enseignant à l'autre. Le tableau suivant illustre uniquement les déclarations des instructeurs quant à leur maitrise des langues officielles et nationales.

Tableau 4 : maîtrise des langues par les enseignants interrogés

\begin{tabular}{|l|l|l|l|}
\hline $\begin{array}{l}\text { Langue } \\
\text { Performances }\end{array}$ & Français & Anglais & LM \\
\hline Très Bien (acrolecte) & $30 \%$ & $41,22 \%$ & $24 \%$ \\
\hline Bien (mésolecte) & $50 \%$ & $34,44 \%$ & $14,88 \%$ \\
\hline Assez Bien (basilecte) & $15,12 \%$ & $16,88 \%$ & $54 \%$ \\
\hline Passable-Nul & $4,88 \%$ & $7,44 \%$ & $7,12 \%$ \\
\hline Total & $100 \%$ & $99,98 \%$ & $100 \%$ \\
\hline
\end{tabular}

Les enseignants de L2 du premier degré auraient un niveau inférieur ou égal à celui requis en classe de Première. En effet, ils manipulent approximativement les deux langues officielles : $30 \%$ des professeurs des écoles maîtrisent parfaitement le français contre $41,22 \%$ pour l'Anglais et $24 \%$ pour les LM. Ainsi, l'accent mis sur la pédagogie des langues et l'exploitation des nouvelles approches semble faciliter l'acquisition de la langue transmise. Mais, pour le français, l'anglais et les LN, considérés comme L2, la pédagogie correspond à celle des langues premières et est le plus souvent centrée sur l'enseignant. Et c'est plutôt l'anglais qui tient lieu de L2 pour les formateurs de formateurs francophones; inversement, c'est le français qui a ce statut pour les anglophones.

Environ $50 \%$ des instructeurs interrogés attestent d'une maitrise quasi parfaite du français contre $34,44 \%$ pour l'anglais, et $14,88 \%$ pour les LM. Outre les contenus pédagogiques (oral, écrit, grammaire, compréhension de texte et orthographe, concepts culturels et discursifs liés à la langue), il leur est également assuré une 
correction systématique de toutes leurs erreurs de langue. Ce qui octroie un double travail aux formateurs des enseignants de L2. Moins de $20 \%$ de la population interrogée est inapte à l'enseignement des L2 car ils estiment que leurs compétences en L2 sont faibles.

Ainsi, la formation des élèves maîtres semble plus difficile, car leurs compétences linguistiques les ne préparent pas à assumer la fonction de formateurs en L2. Les ENIET et les ENIEG n'enseignent qu'une seule L2, le français aux anglophones et l'anglais aux francophones. Mais quel que soit le milieu ou la région, une des deux langues est délaissée au cours de la formation initiale.

Les formateurs de formateurs ne parviennent pas à transmettre des acrolectes et des compétences linguistiques équivalents au cycle de formation initiale pour des raisons multiples comme la maîtrise approximative des langues à véhiculer et le niveau très bas des élèves professeurs en langues nationales (un désavantage pour le projet ELANAfrique $^{6}$ ). En 2012, d'après les sondés, près de $92,88 \%$ d'élèves professeurs du premier cycle manipulent aisément leurs/les LM. Aussi, est-il espéré d'eux une transmission des langues qui allierait compétence, performance et approche pédagogique adéquate.

\section{Formation initiale et actualisation des performances distinctives}

Afin d'optimiser les compétences préalables des élèves enseignants, de recycler les instituteurs et les professeurs en exercice et adopter une approche d'enseignement appropriée aux langues secondes, il faut tenir compte des contenus, des programmes et des objectifs d'enseignement des L2. Aussi, la formation initiale aurait pour but de :

- de remettre en évidence la place cruciale de la compétence en langues dans la réussite scolaire et sociale ;

- de reconstituer l'unité de la compétence langagière, fragmentée en différentes matières scolaires pour le français et l'anglais et de créer ou de renforcer les compétences en langues nationales ;

- d'apprendre et d'utiliser les langues dans sur le terrain, dans des espaces de communication pour contribuer à sa propre formation ;

51 Quatre facteurs participeraient à l'actualisation des compétences: l'adoption des nouvelles approches participatives dans l'enseignement des L2, l'analyse contrastive entre français/ anglais et camfranglais/pidgin-english, et langues maternelles camerounaises, la référence initiale aux compétences premières des futurs enseignants avant le transit progressif vers de nouvelles compétences, révision des programmes dans cette optique.

$52 \mathrm{Au}$ cours des échanges formatifs et évaluatifs, les performances distinctives de ses différentes langues en présence au Cameroun, entre les discours des élèves et des enseignants, sont développées.

53 A) Eléments pour une formation initiale : Les compétences que les apprenants sont censés acquérir en fin de formation sont les suivantes :

- dialogue en français/anglais/LN châtié en prenant soin de clarifier les implicites, d'insister sur les manipulations syntaxiques et les interférences entre le camfranglais, le pidgin et les langues secondes à transmettre ; 
- dialogue en utilisant deux codes : langues locales et langues officielles; les situations contextuelles incitent les enseignants à immerger les apprenants dans la culture du terroir et d'ailleurs ;

- cours de conjugaison, règles de grammaire et d'orthographe

- rédaction des textes courts (la lettre, les demandes d'emploi, le curriculum vitae, etc.)

- lecture et compréhension de textes sur la base des livres et ouvrages illustrés

- adaptation à tous les contextes et situations de communication écrite et orale.

Pour les futurs enseignants de L2, il s'agirait plutôt de :

- la compréhension des différentes approches et méthodes pédagogiques qui conduiront à l'adoption de l'approche la plus adéquate à la classe enseignée ;

- l'appropriation des approches récentes, notamment l'approche actionnelle ;

- l'emploi des NTIC dans les activités de transmission des langues secondes ;

- l'utilisation des manuels et outils d'enseignement de L2 appropriés.

En tête de liste des éléments de la formation initiale, figurent prioritairement la communication orale et écrite, la maitrise de la grammaire, de l'orthographe, de la rhétorique, de la pragmatique, des approches d'enseignement et les NTIC ${ }^{7}$.

B) Approches pédagogiques pour la formation : plusieurs approches sont mises à la disposition des apprenants. Elles participent à l'expression: l'approche participative, l'approche par compétence, l'approche culturelle et audiovisuelle ainsi que l'approche actionnelle.

La figure ci-après illustre les éléments proposés pour utiliser de manière complémentaire les approches didactiques correspondant à l'enseignement des langues :

Figure 2 : approches et implications pédagogiques

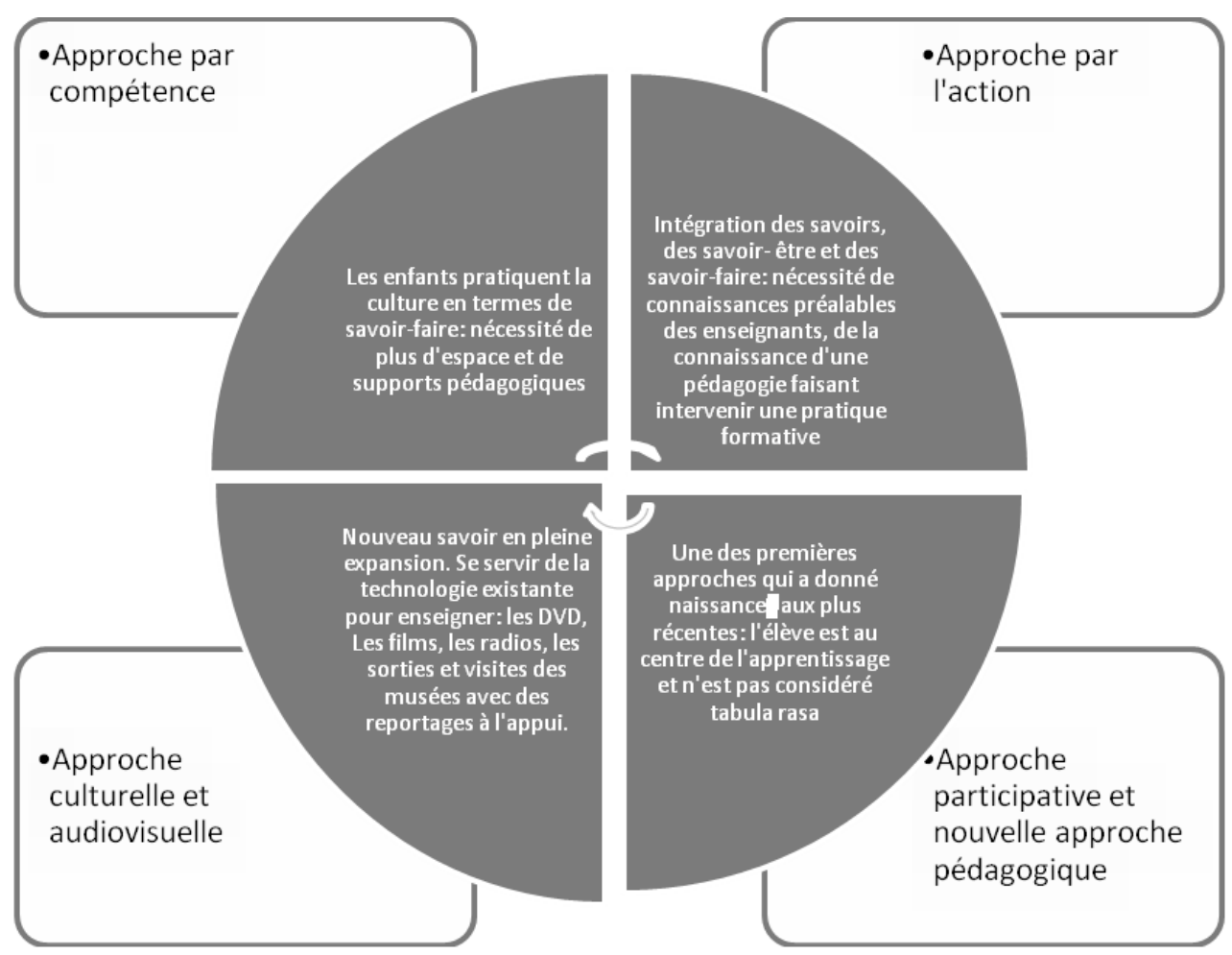


Toutes les approches évoluent et optent pour la participation et la prise en compte des connaissances des élèves pendant les cours. L'approche actionnelle mène aux résultats suivants selon Francis Goullier (2007) :

Figure 3 : illustration de l'approche par l'action par Goullier (2007)

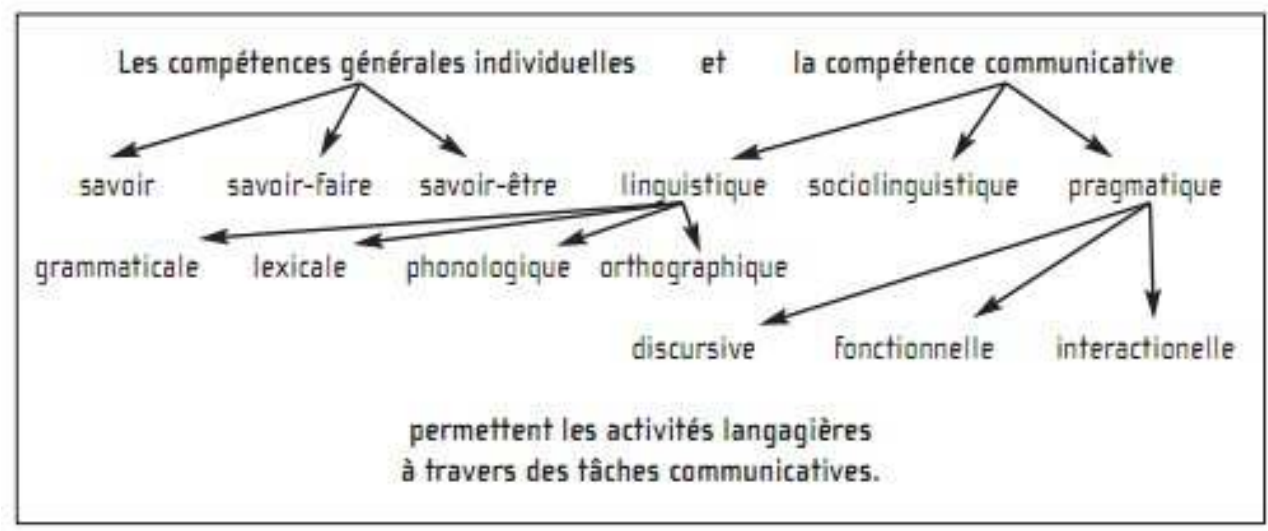

Les compétences à acquérir sont communes aux apprenants et favorisent la communication avec l'extérieur. Les compétences générales individuelles renferment le savoir, le savoir-faire et le savoir-être.

La compétence communicative est l'habileté à utiliser les savoirs, les savoir-faire et les savoir-être pour des activités de communication. Elle implique des connaissances en sociolinguistique, en linguistique et en pragmatique. Aussi, le formateur de L2 doit adapter chaque activité de communication en fonction de ces paramètres.

c) Répartition trimestrielle de quelques contenus: Pour aboutir à une opérationnalisation de l'enseignement des L2, les enseignants doivent répartir quotidiennement, hebdomadairement, mensuellement et trimestriellement les éléments à transmettre. Toutefois, avant de commencer les cours, les enseignants devraient :

- concevoir un test de niveau incluant un examen de conjugaison, de vocabulaire et de grammaire ;

- élaborer une grille de notation dans laquelle il répertoriera les notes en trois groupes : faibles, moyens et forts.

Pour le premier cours, l'enseignant devra commencer par un test oral portant sur les capacités linguistiques des élèves.

Après ce cours, l'instructeur préparera les suivants en fonction des résultats de ce test, en respectant le niveau des apprenants, les besoins des élèves et les objectifs à atteindre. Il faudrait également inclure les exercices de contraste entre les langues communément utilisées par les élèves et la langue cible.

Exemple (4)

Langue de départ : pidgin

*A dõ go skul tude

je prés. partir école aujourd'hui

Je vais à l'école aujourd'hui

Langue cible 1 : anglais

Ai gou tu skul tə'dei 
je prés. à école aujourd'hui

Je vais à l'école aujourd'hui

Langue cible 2 : français

3ə ve a lekol ozurdui

je vais à l'école aujourd'hui

je vais à l'école aujourd'hui

Langue cible 3 : duala

nà mà.lá ó sùkúlù wéng $\bar{\varepsilon}$

je prés.aller à école aujourd'hui

Je vais à l'école aujourd'hui

64 Le contraste se situe au niveau des morphèmes ajoutés ou retirés comme dans

l'exemple b. L'élève devra apprendre la notion du présent simple en anglais, la préposition « to », et la prononciation des diphtongues et des accents.

65 Chaque élément est enseigné en suivant une approche incluant (révision de la leçon, introduction de la conjugaison, leçon proprement dite avec pour base, le pidgin ou le camfranglais, le déroulement avec l'anglais châtié et la phase pratique par des exercices de construction des phrases à l'oral et à l'écrit.).

La deuxième langue-cible cible suit le même procédé que l'anglais. La troisième languecible cible manifeste quelques différences avec le pidgin pris comme élément de départ pour l'enseignement. Il faut insister sur les préfixes verbaux qui établissent la différence entre les temps de la langue nationale. Egalement invoquée, la désignation des différents temps : au lieu du présent de l'indicatif, c'est le présent, au lieu de passé simple, c'est plutôt, le passé récent, etc. Enfin, l'enseignant insistera sur les différences entre les langues dérivationnelles (français, anglais) et les langues agglutinantes (langues bantoues).

67 Aussi les étapes de l'enseignement seront caractérisées par :

- la révision des leçons précédentes

- l'introduction de la leçon en énonçant des phrases en français ou en anglais (n'utiliser un exemple de camfranglais ou de pidgin que si c'est l'élève qui le propose).

- l'écriture au tableau des phrases issues de la/des langue(s) source(s) et à l'opposé de celles-ci les phrases de la langue-cible (phonétiquement et orthographiquement pour faire ressortir les différences et les ressemblances morphosyntaxiques et phonétiques).

- le regroupement des différences et l'enseignement d'un élément grammatical/ communicationnel clé. La suite de la leçon respectera le déroulement habituel d'un cours, c'est-à-dire la phase explicative, des exercices appliqués écrits ou oraux, le transfert par un devoir à faire.

68 La question des compétences linguistiques initiales dans la transmission des langues secondes est primordiale, car elle affecte les performances des élèves et leur adaptation culturelle. Pour cela, il faudrait que les futurs enseignants en tiennent compte au niveau des objectifs d'apprentissage, des approches didactiques et des contenus linguistiques et pédagogiques à transmettre.

69 Soulignons que l'approche actionnelle, centrée sur l'apprenant, de plus en plus utilisée, est compatible avec les compétences communicatives que requièrent les activités d'enseignement qui prônent le recours aux langues secondes pour une meilleure efficacité de leur transmission. 


\section{BIBLIOGRAPHIE}

ADOPO et al (1997). « Le projet -Nord aujourd'hui et demain », dans Langues et éducation en Afrique Noire : Tranel $n^{\circ} 26$.

BILOA, Edmond, (2003). La langue française au Cameroun : analyse linguistique et didactique. Bern : Peter Lang.

BITJAA KODY, Z. D. (2004). La dynamique des langues camerounaises en contact avec le français. Thèse de Doctorat d'Etat. Yaoundé : Université de Yaoundé I.

DIEU, M. ET RENAUD, P. (1983). Atlas linguistique du Cameroun : Inventaire préliminaire. Paris : ACCT, CERDOTOLA et DGRST. Yaoundé.

GOULLIER, Francis, (2007). » Faut-il « appliquer » le Cadre Européen Commun de Référence pour les Langues ». Conférence du 25/09/06 à Reims.

KERBRAT-ORECCHIONI, C. (1986). L'implicite. Paris : Armand Colin.

Le Cadre européen commun de référence pour l'apprentissage, l'enseignement et l'évaluation des langues (CECR)

MESSINA ETHE, J. (2010). Recherche d'une méthodologie de l'enseignement de la culture nationale en milieu plurilingue. Thèse de Doctorat Ph.D. Yaoundé : Université de Yaoundé I.

MINESEC (2007). Enseignement des Langues et Cultures Camerounaises : quelles perspectives ? Séminaire de formation sur les langues et cultures camerounaises, Palais de Congrès.

PUREN, C. (2006). « De l'approche communicative à la perspective actionnelle » dans Le Français dans le Monde $\mathrm{n}^{\circ}$ 347, sept.-oct. 2006, pp. 37-40. Fiche pédagogique correspondante : "Les tâches dans la logique actionnelle", pp. 80-81. Paris : FIPF-CLE international.

SHELL, O. ET WIESEMANN, U. (2000). Guide pour l'alphabétisation en langues africaines. Collection PROPELCA 34. Yaoundé : Université de Yaoundé.

\section{ANNEXES}

Abréviations et acronymes

ALE : Anglais Langue Etrangère

ANACLAC : Association Nationale des Comités de Langues Camerounaises

CECR : Cadre Européen Commun de Référence

DIPES : Diplôme Professionnel de l'Enseignement Secondaire

ÉNIEG : École Normale des Instituteurs de l'Enseignement Général

ENS : École Normale Supérieure

ENSET : École Normale Supérieure de l'Enseignement Technique

FLE : Français Langue Etrangère

LCC : Langues et Cultures Camerounaises 
LM : Langue Maternelle

LN : Langue Nationale

LS (L2) : Langue Seconde

NAP : Nouvelle Approche Pédagogique

PROPELCA : Programme Opérationnel Pour l'Enseignement des Langues au Cameroun

\section{NOTES}

1. Plurilingue : milieu dans lequel il existe plus de deux langues servant à la communication (Op.cit. Bitjaa, 2004).

2. Camfranglais : langue parlée principalement par les jeunes au Cameroun. Cam est mis pour les langues locales camerounaises, fran pour la langue française et anglais pour la langue anglaise. Il est nuancé d'une région à l'autre (Biloa, 2003).

3. Pidgin-english : Langue parlée principalement par les locuteurs des régions du Sud-Ouest et du Nord-Ouest et par les personnes scolarisées en anglais. C'est une langue véhiculaire dans plusieurs pays anglophones comme le Cameroun, le Nigéria, le Ghana, la Sierra Léone. Elle est dérivée essentiellement de l'anglais et a sa propre grammaire.

4. FLE ou ALE : Français Langue Etrangère ou Anglais Langue Etrangère. Il n'ya pas encore de termes pour signifier langue camerounaise étrangère, langue nationale étrangère, etc.

5. Précisons que le Cadre Européen Commun de Référence pour les Langues (2001) insiste sur cette notion: «l'apprenant devient plurilingue et apprend l'interculturalité. Les compétences linguistiques et culturelles relatives à chaque langue sont modifiées par la connaissance de l'autre et contribuent à une prise de conscience interculturelle" (Introduction du CECR. Chapitres 4 et 5).

6. ELAN-Afrique : projet pour l'expérimentation de l'enseignement des langues partenaires du français en Afrique, en l'occurrence les langues africaines. Toutes les compétences linguistiques sont nécessaires.

7. NTIC : Nouvelles Technologies de l'Information et de la Communication. Toutes les universités camerounaises initient leurs étudiants aux NTIC.

\section{RÉSUMÉS}

Le Cameroun, pays d'Afrique centrale, compte près de 248 langues régionales, dont deux langues officielles, à savoir le français et l'anglais. Les Écoles Normales d'Instituteurs et les Écoles Normales Supérieures forment des futurs professeurs de français, d'anglais et de langues régionales. Les variantes créolisées de ces langues, le camfranglais et le pidgin-english, altèrent les performances et les compétences communicatives langagières de l'ensemble des apprenants : élèves, étudiants et futurs enseignants. Notre contribution autour de l'impact du camfranglais et du pidgin-english sur les langues officielles (français, anglais) et régionales, traite des sessions de recyclage, aux remises à niveau et aux activités d'enseignement et d'apprentissage des élèves professeurs de langues secondes et étrangères, à la lumière des nouvelles méthodes 
d'enseignement des langues étrangères, principalement en Europe (CECRL). Cet article ambitionne, ainsi, la contribution à l'amélioration des compétences initiales des futurs enseignants de L2, et par voie de conséquence, à celle des performances de leurs (futurs) élèves.

Cameroon, a country of Central Africa, counts about 248 regional languages among which two official languages known as French and English. The Teachers' Training Colleges of Primary school teachers and the Advance Teachers' Training Colleges train future teachers of French, English and Regional languages. The "creolized" variants of these languages, namely Camfranglais and the Pidgin-English, distort the performances and the linguistic communicative competences of all the learners : pupils, students and teachers to be. Taking into account the impact of the Camfranglais and the Pidgin-English on the official languages (French, English) and regional languages in the light of new teaching methods of foreign languages, principally coming from Europe (CECRL), my contribution is interested in recycling sessions, updating skills and the activities of teaching and learning of trainee-teachers of second and foreign languages. Also, this article aims at improving the initial skills of future teachers of L2 and by extension of the performance of their future pupils while focusing the attention on the content, approaches and teaching activities during their initial training in schools.

\section{INDEX}

Keywords : language in Contact, Multilingualism, Camfranglais, Pidgin-English, Foreign Language, Second Language

Mots-clés : langues en contact, plurilinguisme, camfranglais, pidgin-english, langue étrangère, langue seconde

\section{AUTEUR}

\section{JULIA NDIBNU MESSINA ETHE}

Université de Douala 